PROCEEDINGS OF THE

AMERICAN MATHEMATICAL SOCIETY

Volume 126, Number 1, January 1998, Pages 145-152

S 0002-9939(98)04212-9

\title{
ON SOLVABILITY OF SECOND-ORDER STURM-LIOUVILLE BOUNDARY VALUE PROBLEMS AT RESONANCE
}

\author{
DONG YUJUN
}

(Communicated by Hal Smith)

\begin{abstract}
In this paper, based on of the concept $q_{0} \in H_{0}(p,(0,1), \alpha, \beta)$, which is a generalized form of the first resonant point $\pi^{2}$ to the Picard problem $x^{\prime \prime}+\lambda x=0, x(0)=x(1)=0$, we study the solvability of second-order Sturm-Liouville boundary value problems at resonance $\left(p(t) x^{\prime}\right)^{\prime}+q_{0}(t) x+$ $g(t, x)=h(t), x(0) \cos \alpha-p(0) x^{\prime}(0) \sin \alpha=0, x(1) \cos \beta-p(1) x^{\prime}(1) \sin \beta=$ 0 , and improve the previous results about problems $x^{\prime \prime}+\pi^{2} x+g(t, x)=$ $h(t), x(0)=x(1)=0$ derived by Chaitan P. Gupta, R. Iannacci and M. N. Nkashama, and Ma Ruyun, respectively.
\end{abstract}

\section{INTRODUCTION AND MAIN RESULTS}

Consider the Sturm-Liouville boundary value problem

$$
\begin{gathered}
\left(p(t) x^{\prime}\right)^{\prime}+q_{0}(t) x+g(t, x)=h(t), \\
x(0) \cos \alpha-p(0) x^{\prime}(0) \sin \alpha=0, \\
x(1) \cos \beta-p(1) x^{\prime}(1) \sin \beta=0,
\end{gathered}
$$

where $\alpha, \beta \in R$ are fixed with $0 \leq \alpha<\pi, 0<\beta \leq \pi ; p:[0,1] \rightarrow R$ is a positive absolutely continuous function; $q_{0} \in H_{0}(p,(0,1), \alpha, \beta)$ (here we borrow notation from [1], that is, $q_{n} \in H_{n}(p,(0,1), \alpha, \beta)$ for some nonnegative integer if and only if $q_{n} \in L^{\infty}(0,1)$ and the problem

$$
\left(p(t) x^{\prime}\right)^{\prime}+q_{n}(t) x=0
$$

and (1.2), (1.3) has a nontrivial solution $x=u_{n}(t)$ with exactly $n$ zeros on $\left.(0,1)\right)$; $h \in L^{1}(0,1) ; g:[0,1] \times R \rightarrow R$ is a Caratheodory function, i.e., $g(\cdot, x)$ is measurable on $(0,1)$ for each $x \in R, g(t, \cdot)$ is continuous on $R$ for a.e. $t \in(0,1)$ and for any constant $r>0$ there exists a function $m_{r} \in L^{1}(0,1)$ such that

$$
|g(t, x)| \leq m_{r}(t)
$$

for a.e. $t \in(0,1)$ and all $x \in R$ with $|x| \leq r$.

A function $x \in W^{2,1}(0,1)$ is said to be a solution of (1.1)-(1.3) if $x$ satisfies relations $(1.1)-(1.3)$.

For $a, b \in L^{1}(0,1)$, we denote $a<b$ if and only if $a(t) \leq b(t)$ for a.e. $t \in(0,1)$ and $a(t)<b(t)$ on a subset of $(0,1)$ with positive measure.

Now we state the main result of this paper; the proof follows in the next section.

Received by the editors May 9, 1996.

1991 Mathematics Subject Classification. Primary 34B15.

(C)1998 American Mathematical Society 
Theorem 1. Assume that

i)

$$
g(t, x) x \geq 0 \text { for }|x| \geq r_{0}, \text { a.e. } t \in(0,1)
$$

where $r_{0} \geq 0$ is fixed.

$$
\varlimsup_{|x| \rightarrow \infty} g(t, x) / x \leq \Delta q(t)
$$

uniformly for a.e. $t \in(0,1)$, where $\Delta q \in L^{\infty}(0,1)$ such that

$$
0 \leq \Delta q<q_{1}-q_{0}
$$

with some $q_{1} \in H_{1}(p,(0,1), \alpha, \beta)$.

iii) There exists a constant $\rho>0$ such that

$$
\int_{0}^{1} g\left(t, x_{-}\right) u_{0}(t) d t \leq \int_{0}^{1} h(t) u_{0}(t) d t \leq \int_{0}^{1} g\left(t, x_{+}\right) u_{0}(t) d t
$$

where $u_{0}(t)>0$ on $(0,1)$ satisfies (1.4), (1.2), (1.3) with $n=0$ and

$$
\begin{aligned}
& x_{+} \in\left\{x \in W^{2,1}(0,1) \mid x \text { satisfies }(1.2),(1.3) \text { and } x(t) \geq \rho u_{0}(t) \text { for } t \in[0,1]\right\}, \\
& x_{-} \in\left\{x \in W^{2,1}(0,1) \mid x \text { satisfies }(1.2),(1.3) \text { and } x(t) \leq-\rho u_{0}(t) \text { for } t \in[0,1]\right\} .
\end{aligned}
$$

Then problem (1.1), (1.2), (1.3) has at least one solution.

The results in this paper are inspired by and improve upon the ones given by Chaitan P. Gupta [2] , R. Iannacci and M. N. Nkashama [3], and Ma Ruyun [4], where they discussed the solvability of boundary value problems at resonance

$$
\begin{array}{r}
x^{\prime \prime}+\pi^{2} x+g(t, x)=h(t), \\
x(0)=x(1)=0 .
\end{array}
$$

In those papers, it was necessary to assume that

$$
\varlimsup_{|x| \rightarrow \infty} g(t, x) / x \leq \Gamma(t)
$$

uniformly for a.e. $t \in(0,1)$ and

$$
\Gamma<3 \pi^{2} .
$$

Problem (1.10), (1.11) has not yet been addressed in the case when (1.12)-(1.13) are not satisfied. This was because that in order to prove the boundedness of the solutions for auxiliary equations, the authors $[2,3,4]$ used Fourier series (since $\{\sin n \pi t\}_{n=1}^{\infty}$ forms a complete orthonormal sequence for $\left.L^{2}(0,1)\right)$ and $\pi^{2}+3 \pi^{3}=$ $(2 \pi)^{2}$ is exactly the second eigenvalue of problem $x^{\prime \prime}+\lambda x=0, x(0)=x(1)=0$. However, such problems are discussed in Theorem 1. In the proof, by making use of the functional analysis method, we unify unbounded solutions of the auxiliary nonlinear equations and nontrivial solutions of the relative linear homogeneous equation formed with the growth control function $\Gamma$ and the first resonant point $\pi^{2}$. By this method we not only discuss the case when (1.12)-(1.13) are not satisfied but also improve the previous results $[2,3,4]$ even when (1.12)-(1.13) are satisfied. In fact, in the assumptions of Theorem 1 , let $p=1, \alpha=0, \beta=\pi$; we have the following 
Corollary 1. Assume that

i) $g(t, x) x \geq 0$ for $|x| \geq r_{0}$, a.e. $t \in(0,1)$ where $r_{0} \geq 0$ is fixed.

ii) $\varlimsup_{|x| \rightarrow \infty} g(t, x) / x \leq \Delta q(t)$ uniformly for a.e. $t \in(0,1)$ where $\Delta q \in L^{\infty}(0,1)$ such that

$$
0 \leq \Delta q<q_{1}-q_{0}
$$

with $q_{i} \in L^{\infty}(0,1)$ such that

$$
\begin{aligned}
& x^{\prime \prime}+q_{i}(t) x=0, \\
& x(0)=x(1)=0
\end{aligned}
$$

has a nontrivial solution $x=u_{i}(t)$ with exactly $i$ zeros on $(0,1), i=0,1$, and we assume that $u_{0}(t)>0$ on $(0,1)$.

iii) There exists a constant $\rho>0$ such that

$$
\int_{0}^{1} g\left(t, x_{-}\right) u_{0}(t) d t \leq \int_{0}^{1} h(t) u_{0}(t) d t \leq \int_{0}^{1} g\left(t, x_{+}\right) u_{0}(t) d t
$$

where

$$
\begin{aligned}
& x_{+} \in\left\{x \in W^{2,1}(0,1) \mid x(0)=x(1)=0 \text { and } x(t) \geq \rho u_{0}(t) \text { for } t \in[0,1]\right\}, \\
& x_{-} \in\left\{x \in W^{2,1}(0,1) \mid x(0)=x(1)=0 \text { and } x(t) \leq-\rho u_{0}(t) \text { for } t \in[0,1]\right\} .
\end{aligned}
$$

Then problem

$$
\begin{aligned}
& x^{\prime \prime}+q_{0}(t) x+g(t, x)=h(t), \\
& x(0)=x(1)=0
\end{aligned}
$$

has at least one solution.

Remarks. 1. Corollary 1 contains [2, Theorem 2.4], [3, Theorem 1] and [4, Theorem 1] as special cases when $q_{0}=\pi^{2}, q_{1}=4 \pi^{2}$ since in $[2,3,4]$ the authors assumed that $m_{r}$, which satisfies $(1.5)$, is an element of $L^{2}(0,1)$.

2. If $q_{0}(t)=\pi^{2}, \pi^{2}<A \leq \pi^{2}+\Delta q(t)$ and $\int_{0}^{1} \Delta q(t) d t<A-\pi^{2}+4 \sqrt{A} \operatorname{ctg} \frac{\sqrt{A}}{4}$, then condition (1.14) is valid from [1, Corollary 8].

3. If $q_{0}=\pi^{2}, \Delta q \in L^{\infty}(0,1)$ such that $0 \leq \Delta q$ and

$$
\int_{0}^{1}\left(\pi^{2}+\Delta q(t)\right)^{1 / 2} d t+\frac{1}{4} \int_{0}^{1}\left|d \ln \left(\pi^{2}+\Delta q(t)\right)\right|<2 \pi
$$

then condition (1.14) is satisfied from [1, Corollary 9].

Example 1. Let $q_{0}(t)=\pi^{2}, g(t, x)=\Gamma(t) x \sin ^{2} x$ with $\Gamma(t)=\frac{\pi^{2}}{t_{1}^{2}}-\pi^{2}$ for $t \in$ $\left(0, t_{1}\right), \Gamma(t)=0$ for $t \in\left(t_{1}, 1\right)$ and $\int_{0}^{1} h(t) \sin \pi t d t=0$. Then from Corollary 1 problem (1.10) (1.11) has a solution since, letting $\bar{\Gamma}(t)=\frac{\pi^{2}}{t_{1}^{2}}-\pi^{2}$ for $t \in\left(0, t_{1}\right)$ and $\bar{\Gamma}(t)=\frac{\pi^{2}}{\left(1-t_{1}\right)^{2}}-\pi^{2}$ for $t \in\left(t_{1}, 1\right)$, we have that $\Gamma<\bar{\Gamma}$ and $\pi^{2}+\bar{\Gamma} \in H_{1}(1,(0,1), 0, \pi)$. But for $t \in\left(0, t_{1}\right), \Gamma(t) \rightarrow+\infty$ as $t_{1} \rightarrow 0^{+}$, hence this case cannot be solved by $[2,3,4]$.

Example 2. Let $q_{0}(t)=\frac{\pi^{2}}{4 t_{1}^{2}}$ for $t \in\left(0, t_{1}\right), q_{0}(t)=\frac{\pi^{2}}{4\left(1-t_{1}\right)^{2}}$ for $t \in\left(t_{1}, 1\right), \Delta q(t)=$ $\frac{3 \pi^{2}}{4 t_{1}^{2}}$ for $t \in\left(0, t_{1}\right), \Delta q(t)=0$ for $t \in\left(t_{1}, 1\right), g(t, x)=\Delta q(t) x \sin ^{2} x, u_{0}(t)=\sin \frac{\pi}{2 t_{1}} t$ for $t \in\left(0, t_{1}\right), u_{0}(t)=\sin \frac{\pi}{2\left(1-t_{1}\right)}(1-t)$ for $t \in\left(t_{1}, 1\right)$, and $h \in L^{1}(0,1)$ such that $\int_{0}^{1} u_{0}(t) h(t) d t=0$. Then problem (1.15) (1.16) has at least one solution from Corollary 1. 
From Theorem 1 , let $p=1, \alpha=\beta=\frac{\pi}{2}, q_{0} \in H_{0}\left(1,(0,1), \frac{\pi}{2}, \frac{\pi}{2}\right)$. We have

Corollary 2. Assume that

i) $g(t, x) x \geq 0$ for $|x| \geq r_{0}$, a.e. $t \in(0,1)$ where $r_{0} \geq 0$ is fixed.

ii) $\varlimsup_{|x| \rightarrow \infty} g(t, x) / x \leq \Delta q(t)$ uniformly for a.e. $t \in(0,1)$, where $\Delta q \in L^{\infty}(0,1)$ such that

$$
0 \leq \Delta q<q_{1}-q_{0}
$$

with $q_{i} \in L^{\infty}(0,1)$ such that

$$
\begin{aligned}
& x^{\prime \prime}+q_{i}(t) x=0, \\
& x^{\prime}(0)=x^{\prime}(1)=0
\end{aligned}
$$

has a nontrivial solution $x=u_{i}(t)$ with exactly $i$ zeros on $(0,1), i=0,1$, and $u_{0}(t)>0$ for $t \in[0,1]$.

iii) There exists a constant $\rho>0$ such that

$$
\int_{0}^{1} g\left(t, x_{-}\right) u_{0}(t) d t \leq \int_{0}^{1} h(t) u_{0}(t) d t \leq \int_{0}^{1} g\left(t, x_{+}\right) u_{0}(t) d t
$$

where $x_{-}<-\rho, x_{+}>\rho$ for $x_{-}, x_{+} \in W^{2,1}(0,1)$ with $x_{-}^{\prime}(0)=x_{-}^{\prime}(1)=x_{+}^{\prime}(0)=$ $x_{+}^{\prime}(1)=0$.

Then problem

$$
\begin{aligned}
& x^{\prime \prime}+q_{0}(t) x+g(t, x)=h(t), \\
& x^{\prime}(0)=x^{\prime}(1)=0
\end{aligned}
$$

has at least one solution.

Remarks. 1. In Corollary 2, letting $q_{0}=0, q_{1}=\pi^{2}$, we get a generalized form of [3, Theorem 2].

2. In Theorem 1 , by specifying $p, \alpha, \beta$ we may obtain other new special cases.

\section{Proof of Theorem 1}

This section is devoted to the proof of Theorem 1. To this end, let us first introduce some lemmas.

Lemma 1. Assume that $a \in L^{\infty}(0,1)$ and $b \in H_{1}(p,(0,1), \alpha, \beta)$ with $a<b$. Then there exist a number $\delta>0$ and $\bar{b} \in H_{1}(p,(0,1), \alpha, \beta)$ such that $a+\delta<\bar{b}$.

Proof. This proof follows directly from Lemmas 2-4 and Proposition 4 of [1]. Since the reference [1] has not been published yet, let us sketch the proof here.

Consider the initial value problem

$$
\begin{aligned}
& \left(p(t) x^{\prime}\right)^{\prime}+q(t) x=0, \\
& x(0)=\sin \alpha, p(0) x^{\prime}(0)=\cos \alpha .
\end{aligned}
$$

Assume that $x=x(t)$ is a solution of (2.1) (2.2), and introduce the famous Prüfer transformation

$$
\varphi=\arctan \frac{x(t)}{p(t) x^{\prime}(t)}
$$

Then we have

$$
\varphi^{\prime}=\frac{1}{p(t)} \cos ^{2} \varphi+q(t) \sin ^{2} \varphi, \quad \varphi(0)=\alpha
$$


Denote the solution of (2.4) by $\varphi=\varphi(t, q)$; then, similar to the proof of [5, Chapter XI, Lemma 3.1], we can prove that $q$ in $H_{1}(p,(0,1), \alpha, \beta)$ if and only if the unique solution of $(2.4)$ satisfies $\varphi(1, q)=\pi+\beta$. Hence $\varphi(1, a)<\varphi(1, b)=\pi+\beta$. In view of [5, P. 4, Theorem 2.4] and [6, Chapter 8, Theorem 2.1], there exists $\delta>0$ such that $\varphi(1, a+\delta)<\varphi(1, a+2 \delta)=\pi+\beta$, and obviously $a+2 \delta \in H_{1}(p,(0,1), \alpha, \beta)$.

Lemma 2. Assume that $q_{i} \in H_{i}(p,(0,1), \alpha, \beta), i=0,1, q \in L^{\infty}(0,1)$ satisfying $q_{0}<q<q_{1}$. Then problem (2.1) (1.2) (1.3) has no nontrivial solution; moreover problem

$$
\left(p(t) x^{\prime}\right)^{\prime}+q(t) x=h(t)
$$

and (1.2) (1.3) have a unique solution for $h \in L^{1}(0,1)$.

Proof. Similar to [5, P. 337, Theorem 4.1 (iv)].

Lemma 3. Assume that $x \in C^{1}(0,1)$ satisfying condition (1.2) (1.3). Then there exists a constant $C$ depending only on $u_{0}$ such that

$$
|x(t)| \leq C|x|_{1} u_{0}(t), \quad t \in[0,1]
$$

where $|\cdot|_{1}$ is the norm of the usual Banach space $C^{1}(0,1)$ and $u_{0}(t)>0$ on $(0,1)$ is a solution of (1.4) (1.2) (1.3) with $n=0$.

Proof. Since $u_{0}(t)>0$ on $(0,1)$, we need only consider the case when $t=0$ or $t=1$. If $\alpha=0$, then $u_{0}(0)=0$, but $u_{0}(t)$ is a nontrivial solution of $(2.1)$; it follows that $u_{0}(0) \neq 0$. Hence

$$
\left|\frac{x(t)}{u_{0}(t)}\right|=\left|\frac{x(t)-x(0)}{u_{0}(t)-u_{0}(0)}\right|=\left|\frac{x^{\prime}(\xi)}{u_{0}^{\prime}(\xi)}\right| \leq \frac{2}{\left|u_{0}^{\prime}(0)\right|}|x|_{1}
$$

for sufficiently small $t>0$. The proof is complete.

Proof of Theorem 1. From Lemma 1 and assumption ii) there exist a number $\delta>0$ and $\bar{q}_{1} \in H_{1}(p,(0,1), \alpha, \beta)$ such that

$$
q_{0}+\Delta q+2 \delta<\bar{q}_{1} .
$$

In view of assumption ii) there exists a constant $r_{1}>r$ such that

$$
g(t, x) / x \leq \Delta q(t)+\delta \text { for a.e. } t \in(0,1) \text {, and all } x \in R \text { with }|x| \geq r_{1} .
$$

Denote

$$
L: W_{0}^{2,1}(0,1) \subset C^{1}(0,1) \rightarrow L^{1}(0,1), x \rightarrow\left(p(t) x^{\prime}(t)\right)^{\prime}+\left[q_{0}(t)+\delta\right] x(t),
$$

where $W_{0}^{2,1}(0,1) \triangleq\left\{x \in W^{2,1}(0,1): x\right.$ satisfies conditions (1.2) (1.3) $\}$. Then from Lemma 2 the operator $L$ is one-to-one, onto and obviously continuous.

It follows that the inverse of $L$ exists, $L^{-1}: L^{1}(0,1) \rightarrow W_{0}^{2,1}(0,1)$, is continuous and $L^{-1}: L^{1}(0,1) \rightarrow C^{1}(0,1)$ is compact since $W^{2,1}(0,1)$ is compactly imbedded into $C^{1}(0,1)$.

Define $G: C^{1}(0,1) \rightarrow L^{1}(0,1)$ by

$$
G x=\delta x(t)-g(t, x(t))+h(t) .
$$

Then $G$ is a continuous operator and problem (1.1) (1.2) (1.3) can be written in the equivalent form

$$
L x=G x .
$$


Consider the auxiliary equation

$$
L x=\lambda G x, \quad \lambda \in(0,1) .
$$

In view of the Leray-Schauder continuation theorem and considering the compactness of $L^{-1}$ from $L^{1}(0,1)$ to $C^{1}(0,1)$, in order to prove that $(2.9)$ has a solution, we need only demonstrate that the possible solution of $(2.10)_{\lambda}$ is bounded. If not, there exist sequences $\left\{x_{n}\right\} \subseteq \operatorname{dom} L$ and $\left\{\lambda_{n}\right\} \subseteq(0,1)$ such that $\left|x_{n}\right|_{1} \rightarrow \infty$ as $n \rightarrow \infty$ and

$$
L x_{n}=\lambda_{n} G x_{n} .
$$

Denote $y_{n}=x_{n} /\left|x_{n}\right|_{1}$; then (2.11) becomes

$$
L y_{n}=\lambda_{n}\left|x_{n}\right|_{1}^{-1} G x_{n}=\lambda_{n} \delta y_{n}-\lambda_{n}\left|x_{n}\right|_{1}^{-1} g\left(t, x_{n}(t)\right)+\lambda_{n}\left|x_{n}\right|_{1}^{-1} h(t) .
$$

Define

$$
\mu_{n}(t)= \begin{cases}g\left(t, x_{n}(t)\right) / x_{n}(t) & \text { for }\left|x_{n}(t)\right| \geq r_{1}, \\ 0 & \text { for }\left|x_{n}(t)\right|<r_{1} .\end{cases}
$$

We have from (2.7) that

$$
0 \leq \mu_{n}(t) \leq \Delta q(t)+\delta \quad \text { for a.e. } t \in(0,1)
$$

and hence we assume that

$$
\mu_{n} \rightarrow \mu_{0}
$$

in $L^{2}(0,1)$ for some $\mu_{0} \in L^{2}(0,1)$ such that

$$
0 \leq \mu_{0}(t) \leq \Delta q(t)+\delta \text { for a.e. } t \in(0,1) .
$$

Denote

$$
f_{n}(t)=g\left(t, x_{n}(t)\right)-\mu_{n}(t) x_{n}(t)
$$

then (2.11) can be written in the form

$$
L y_{n}=\lambda_{n} \delta y_{n}-\lambda_{n} \mu_{n}(t) y_{n}+\lambda_{n}\left|x_{n}\right|_{1}^{-1}\left[h(t)-f_{n}(t)\right] .
$$

In view of (2.13), $f_{n}(t)$ vanishes if $\left|x_{n}(t)\right| \geq r_{1}$, and hence the right hand side of $(2.16)$ must be bounded in $L^{1}(0,1)$ for $n=1,2, \ldots$ Using the compactness of $L^{-1}: L^{1}(0,1) \rightarrow C^{1}(0,1)$, we may assume that $y_{n} \rightarrow y_{0}$ in $C^{1}(0,1)$ for some $y_{0} \in C^{1}(0,1)$, and $\lim _{n \rightarrow \infty} \lambda_{n}=\lambda_{0} \in[0,1]$. Therefore, the right hand side of (2.16) converges weakly to $\lambda_{0} \delta y_{0}-\lambda_{0} \mu_{0} y_{0}$ in $L^{1}(0,1)$. Again using the compactness of $L^{-1}: L^{1}(0,1) \rightarrow C^{1}(0,1)$, we have

$$
L y_{0}=\lambda_{0}\left(\delta-\mu_{0}\right) y_{0},
$$

or equivalently

$$
\left(p(t) y_{0}^{\prime}\right)^{\prime}+\left[q_{0}(t)+\left(1-\lambda_{0}\right) \delta+\lambda_{0} \mu_{0}(t)\right] y_{0}=0 .
$$

From (2.6), (2.15), $\left|y_{0}\right|_{1}=1$ and Lemma 2 , we have that $\left(1-\lambda_{0}\right) \delta+\lambda_{0} \mu_{0}=0$, and hence

$$
\left(p(t) y_{0}^{\prime}\right)^{\prime}+q_{0}(t) y_{0}=0
$$

from which it follows that

$$
y_{0}= \pm u_{0}
$$

where we assume that $\left|u_{0}\right|_{1}=1$. 
Denote

$$
\bar{y}_{n}(t)=k_{n} u_{0}(t), \quad \tilde{y}_{n}=y_{n}(t)-\bar{y}_{n}(t)
$$

where

$$
k_{n}=\left[\int_{0}^{1} u_{0}^{2}(t) d t\right]^{-1} \int_{0}^{1} y_{n}(t) u_{0}(t) d t
$$

Then

$$
\int_{0}^{1} \tilde{y}_{n}(t) u_{0}(t) d t=0
$$

and hence

$$
k_{n}^{2} \int_{0}^{1} u_{0}^{2}(t) d t+\int_{0}^{1} \tilde{y}_{n}^{2}(t) d t=\int_{0}^{1} y_{n}^{2}(t) d t \leq 1 .
$$

We may assume that

$$
k_{n} \rightarrow \bar{k} \in R
$$

By (2.18) if $y_{0}=u_{0}$, from (2.19) we have $\bar{k}=1$ and

$$
\tilde{y}_{n} \rightarrow 0 \text { in } C^{1}(0,1) \text {. }
$$

From Lemma 3, there exists $N>0$ such that

$$
\left|x_{n}\right|_{1}>4 \rho, \quad k_{n}>\frac{1}{2}, \quad\left|\tilde{y}_{n}(t)\right| \leq \frac{1}{4} u_{0}(t) \quad \text { for } t \in[0,1], n \geq N,
$$

whence it follows that

$$
x_{n}(t)=\left|x_{n}\right|_{1} y_{n}(t) \geq \rho u_{0}(t) \quad \text { for } t \in[0,1], n \geq N .
$$

Taking the inner product (in $L^{2}(0,1)$ ) of $(2.12)$ with $u_{0}$, considering $(2.17)$ and $y_{0}=u_{0}$, and $y_{n}$ satisfying (1.2) (1.3), we obtain

$$
\begin{aligned}
& \left(1-\lambda_{n}\right) \delta k_{n} \int_{0}^{1} u_{0}^{2}(t) d t+\lambda_{n}\left|x_{n}\right|_{1}^{-1} \int_{0}^{1} g\left(t, x_{n}(t)\right) u_{0}(t) d t \\
& =\lambda_{n}\left|x_{n}\right|_{1}^{-1} \int_{0}^{1} h(t) u_{0}(t) d t
\end{aligned}
$$

from which it follows from (2.20) that

$$
\int_{0}^{1} g\left(t, x_{n}(t)\right) u_{0}(t) d t<\int_{0}^{1} h(t) u_{0}(t) d t, \quad n \geq N,
$$

a contradiction with assumption iii) and (2.21).

In a way similar to the case $y_{0}=u_{0}$ we can also obtain a contradiction in the case $y_{0}=-u_{0}$. Therefore, the solutions of $(2.10)_{\lambda}$ are bounded and the proof is complete.

\section{ACKnowledgement}

The author would like to express his sincere thanks to Professor Hal L. Smith and the referee for their great patience and conscientiousness shown in the refereeing of the manuscript. 


\section{REFERENCES}

[1] Dong Yujun, On equivalent conditions for the solvability of equation $\left(p(t) x^{\prime}\right)^{\prime}+f(t, x)=h(t)$ satisfying linear boundary value conditions with $f$ restricted by linear growth conditions, to appear in J. Math. Anal. Appl.

[2] Chaitan P. Gupta, Solvability of a boundary value problem with the nonlinearity satisfying a sign condition, J. Math. Anal. Appl. 129 (1988), 482-492. MR 89a:34024

[3] R. Iannacci and M. N. Nkashama, Nonlinear two point boundary value problems at resonance without Landesman-Lazer condition, Proc. Amer. Math. Soc. 106 (1989), 943-952. MR 90f:34031

[4] Ma Ruyun, Solvability of a class of semilinear two-point boundary value problems at resonance, Acta Mathematica Sinica (in Chinese) 36 (1993), 99-105. MR 94g:34031

[5] Philip Hartman, Ordinary Differential Equations, Second Edition, 1982, Birkhäuser, Boston, Basel, Stuttgart. MR 83e:34002

[6] E. A. Coddington and N. Levinson, Theory of Ordinary Differential Equations, International Series in Pure and Applied Mathematics, 1955. MR 16:1022b

Institute of Mathematics, Jilin University, Changchun, Jilin, 130023, People's RePUBLIC OF CHINA 